\title{
Eye Movements Reflect Reasoning with Mental Images but Not with Mental Models in Orientation Knowledge Tasks
}

\author{
Jan Frederik Sima, Maren Lindner, Holger Schultheis, and Thomas Barkowsky
}

\author{
SFB/TR 8 Spatial Cognition, Universität Bremen, Enrique-Schmidt-Str. 5, \\ 28359 Bremen, Germany \\ \{sima,mlindner, schulth, barkowsky\}@sfbtr8.uni-bremen. de
}

\begin{abstract}
This paper presents results showing that eye movements reflect spatial relations in mental images but not in mental models during nearly similar reasoning tasks with directions. These results contribute to the distinction between mental models and mental images based on eye movements. This differentiation may be applied in the field of human-computer interaction and intelligent assistance systems. We conducted two experiments about reasoning with cardinal directions employing three-term series problems in the form of: " $\mathrm{X}$ is southwest of $\mathrm{Z}$; $\mathrm{Y}$ is east of $\mathrm{X}$; as seen from $\mathrm{Z}$, where is $\mathrm{Y}$ ?" The results replicate, to some extent, previous findings about preferred mental models. Additionally, the results indicate that these preferences are susceptible to details of the instructions of the experiment.
\end{abstract}

\section{Introduction}

This paper investigates the connection between reasoning with orientation knowledge and eye movements. We distinguish between two different mental representations that can underlie such a reasoning process, namely visual mental images (Kosslyn, 1994) and spatial mental models (Johnson-Laird, 1983; Tversky, 1993). In addition to previous eye tracking studies, which already found connections between eye movements and mental imagery, this study also explores the connection between eye movements and reasoning based on more abstract representations, that is, spatial mental models. To our knowledge, there are currently no studies that focused on eye movements in connection with spatial mental models. It is the aim of this study to investigate possible differences in eye movements during reasoning based on these different spatial representations. Spatial mental models are assumed to be abstract and amodal representations. In contrast, visual mental images are quasi-pictorial representations containing vivid details. Mental images can be described as resembling, at least to some degree, the experience of actually visually perceiving an object or a scene (Finke 1989). We hypothesize that there are less eye movements reflecting the content of a mental model than that of a mental image. Being able to distinguish these different representations during spatial reasoning tasks potentially provides new possibilities for the field of human computer interaction. For instance, if a computational assistance system is aware of the representation format the human reasoner employs, the system will be in a better position to provide appropriate assistance. 
Following up on the results of Bertel, Schultheis, and Barkowsky (2010), the conducted experiments also aim at exploring possible preferences in human reasoning about orientation relations. In this paper we compare two representation formats for deductive reasoning tasks with cardinal direction knowledge. The tasks are presented as three-term series problems (Johnson-Laird, 1972). Orientation knowledge can be considered a fundamental spatial knowledge type. Other fundamental spatial knowledge types are distance or topology. In this paper we focus on cardinal directions (e.g., north, northeast, east, etc.) as one form of orientation knowledge. Cardinal directions are qualitative relations given in terms of a global, geocentric reference system.

\subsection{Reasoning with Orientation Knowledge in Under-Determined Tasks}

Some reasoning tasks are not fully specified, that is, the given premises describe more than one spatial situation, thus, the problem is under-determined. In reasoning with orientation knowledge it is not possible to infer new orientation relations unambiguously when only orientation, but no distance relations between the entities are provided (Frank, 1996). A mental model, by definition, only represents one single instantiation of the described situation (Johnson-Laird, 1989). Several empirical studies in the field of spatial cognition showed that in the case of under-determined reasoning problems humans tend to construct only one mental model at a time to solve a problem rather than to construct all possible models (Knauff, Rauh, \& Schlieder, 1995; Jahn, Johnson-Laird, \& Knauff, 2005). These mental models, that are constructed primarily, have been termed preferred mental models. It is assumed that preferred mental models are easier to generate and faster to process and that they thus provide an efficient way to reason even with under-determined spatial information. Exploration of preferred mental models can accordingly make an important contribution to the understanding of human spatial reasoning.

Bertel et al. (2010) carried out an experiment to investigate preferred mental models in human deductive reasoning with cardinal directions. They had subjects work on three-term series problems containing two out of eight cardinal directions. An example of such a task would be: $\mathrm{X}$ is southwest of $\mathrm{Z}\left(1^{\text {st }}\right.$ premise $), \mathrm{Y}$ is east of $\mathrm{X}$ ( $2^{\text {nd }}$ premise), as seen from $\mathrm{Z}$, where is $\mathrm{Y}$ ? (question). The given problems were restricted to those in which fictive lines between $X$ and $Z$ and between $Y$ and $X$ form a $45^{\circ}$ (e.g., $\mathrm{X}$ is southwest of $\mathrm{Z}, \mathrm{Y}$ is east of $\mathrm{X}$ ) or a $90^{\circ}$ angle (e.g., $\mathrm{X}$ is south of $\mathrm{Z}, \mathrm{Y}$ is east of $X$ ). Given an eight-sector model of cardinal directions as the basis (see Fig. 1), four (for the $45^{\circ}$ problems) or three (for the $90^{\circ}$ problems) of the eight directions are correct answers; i.e., the reasoning problem is under-determined.

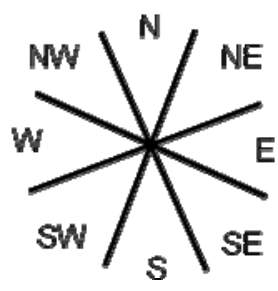

Fig. 1. Eight-sector model of cardinal directions 
Figure 2 shows a taxonomy of possible mental models underlying these correct answers. Based on the subject's answer, the used mental model can be classified. If the distances between the entities are assumed to be equal, model $\boldsymbol{b}$ is constructed to solve the task. The answer would therefore be "southeast". If the distance between X and Z is longer than the distance between $\mathrm{X}$ and $\mathrm{Y}$, model $\boldsymbol{c}$ is used and the according answer would be "south". Model $\boldsymbol{a}$ in which the distance between X and Z is considerably shorter than between $X$ and $Y$ results in the answer "east". On the contrary, if the distance between $\mathrm{X}$ and $\mathrm{Z}$ is considerably longer than the distance between $\mathrm{X}$ and $\mathrm{Y}$, model $\boldsymbol{d}$ is used, which leads to the answer "southwest".

a

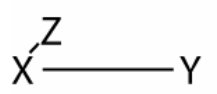

b

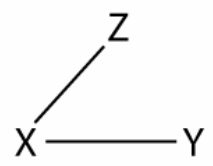

C

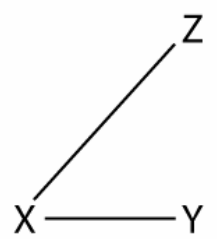

d

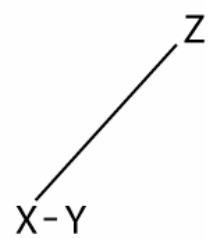

Fig. 2. Different possible mental models to solve the reasoning task " $\mathrm{X}$ is southwest of $\mathrm{Z}$; $\mathrm{Y}$ is east of X; as seen from Z, where is Y?" The triangles of model $\boldsymbol{b}$ and $\boldsymbol{c}$ are considered prototypical and model $\boldsymbol{c}$ is the preferred model as the to be inferred direction is "south" compared to "southeast" in model $\boldsymbol{b}$.

Bertel et al. (2010) hypothesize that there are preferred mental models in reasoning with cardinal directions and that models will be preferred which a) form a prototypical triangle to constitute the problem situation and b) lead to one of the four main cardinal directions, i.e., north, east, south or west, as the inferred direction. Furthermore, property b) is weighted stronger than property a). Figure 2 gives an example of this preference. Thus, these assumptions lead to one hypothesized specific preferred model for each of the reasoning problems under investigation. Figure 3 illustrates two examples for hypothesized preferred mental models of $45^{\circ}$ problems (1 and 2) and two for $90^{\circ}$ problems (3 and 4).
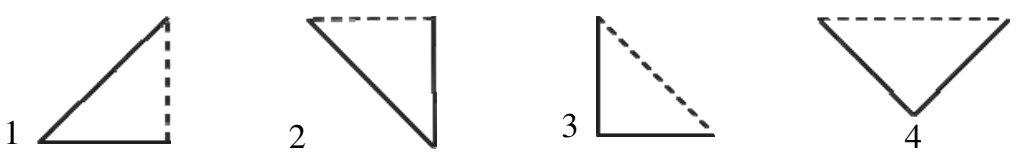

Fig. 3. Examples of hypothesized preferred mental models for $45^{\circ}$ problems (1 and 2$)$ and for $90^{\circ}$ problems (3 and 4)

In the rest of this paper and in particular in the analysis we will use the following terms to distinguish between the possible models. The model that forms a prototypical triangle and leads to a main cardinal direction as the answer will be called "preferred model". The model that only forms a prototypical triangle will by called "prototypical 
model". For $90^{\circ}$ problems there are no prototypical models as those are always also preferred. The other models, which form distorted triangles, will be referred to as "distorted models".

\subsection{Mental Representations and Eye Movements}

It has been argued that eye movements are linked to processes of attentional control (Shepherd, Findlay, \& Hockey, 1986) and that they can be used as an indication of what the subject pays attention to and what he or she is concerned with in a particular task. Furthermore, there is a connection between visual information processing and eye movements as they are linked with visual brain areas by a projection path from extrastriate visual areas via the posterior part of the parietal cortex to regions associated with eye movements (Knauff, 2009). Several studies used eye tracking methodology to investigate mental images and showed a direct relation between eye movements and the processing of spatial relations in an imagined scene (e.g., Johansson, Holsanova, \& Holmqvist, 2006). On the one hand, there are studies that show similarities between eye movements while subjects look at a particular picture and eye movements while these subjects imagine the previously seen picture (Brandt \& Stark, 1997; Laeng \& Teodorescu, 2001). On the other hand, there are experiments tracking the eye movements of subjects during the imagination of a previously unknown scene (Demarais \& Cohen, 1998; Johansson et al., 2006; Ragni, Fangmeier, Bittner, \& Konieczny, 2009; Spivey \& Geng, 2001). In both cases there is clear correspondence between the eye movement and the mental image. There also are studies that show connections between eye movements and spatial relations in acoustically presented reasoning tasks (Demarais \& Cohen, 1998; Ragni et al., 2009).

All these studies either presented the stimuli before the imagery phase or employed stimuli that are very easy to visualize and not abstract. For example, subjects had to imagine a colored fish (Laeng \& Teodorescu, 2001), a forty story building with different actions happening on each floor (Spivey \& Geng, 2001) or a scene rich of details and with many objects and spatial relations (Johansson et al., 2006). The reasoning tasks of Demarais and Cohen (1998) were also designed for easy visualization, e.g., by using household objects arranged in a shelf like "a jar of pickles is below a box of tea bags; the jar of pickles is above a can of coffee; where's the can of coffee?"

It can be assumed that the way the experimental tasks are presented to a subject influences the mental representation used to solve these tasks. According to the definitions of mental images (Finke, 1989; Kosslyn, 1994), we state that if the stimuli are easy to visualize and contain a lot of vivid and visual details, subjects will employ visual mental images. In line with the definition of mental models (Johnson-Laird, 1983), we argue that if the stimuli are abstract and amodal, subjects will employ a non-visual but spatial representation, i.e., a spatial mental model. In order to grade stimuli as being more visual or more abstract, we refer to Schultheis, Bertel, Barkowsky, and Seifert (2007); they defined criteria to classify representations as being rather abstract and spatial or as being more vivid and visual. One criterion is for example exemplarity. That is, the more the stimulus is an example of a known situation (compared to a category or an abstract prototype) the more visual the used representation will be. Our assumptions are in line with the theory of deductive reasoning with mental models and visual mental images of Knauff (2009): 
"Visual brain areas are only involved if the problem information is easy to visualize and when this information must be processed and maintained in visual working memory. A regular reasoning process, however, does not involve visual images but more abstract spatial representations - spatial mental models - held in parietal cortices." (p. 111)

The discussed eye tracking studies mostly state that they investigate mental imagery and all of them used relatively easy-to-visualize stimuli. Additionally, they all reported eye movements according to the spatial relations of the stimuli. This shows that processing of mental images seemingly correlates with according eye movements. However, there seem to be no studies that report eye movements that reflect the spatial relations of spatial mental models. We assume that there is no systematic relation between eye movements and reasoning with spatial mental models. It is one aim of this contribution to test this assumption. Accordingly, we present two eye tracking experiments with similar reasoning tasks for which subjects are assumed to construct mental models or mental images, respectively.

\section{Experiment 1 - Reasoning with Mental Models}

The stimulus materials as well as the hypotheses regarding preferred mental models are as detailed in Bertel et al. (2010). It is the aim of experiment 1 to investigate the connection between eye movements and reasoning based on mental models. The reasoning problems used in this experiment are very abstract and not easy to visualize and it is assumed that subjects will thus use spatial mental models. It is further hypothesized that eye movements are not connected with reasoning based on spatial mental models, and that eye movements will therefore not occur significantly more often according to the directions given in the tasks compared to all other directions.

\subsection{Participants and Materials}

25 undergraduate students of the University of Bremen, 11 male and 14 female, volunteered to take part in the experiment for monetary compensation.

The three-term series problems used in the experiments contained the eight cardinal directions north, northwest, west, southwest, south, southeast, east, and northeast. Each trial is composed of two premises about two cardinal directions between three entities. The third direction relation is to be concluded. The entities are labeled with letters. The following is an example trial: " $\mathrm{X}$ is southwest of $\mathrm{Z}$; $\mathrm{Y}$ is east of $\mathrm{X}$; as seen from $\mathrm{Z}$, where is Y?"

For every problem trial the directions were chosen so that a line between $\mathrm{X}$ and $\mathrm{Z}$ and a line between $\mathrm{Y}$ and $\mathrm{X}$ would form either a $45^{\circ}$ or a $90^{\circ}$ angle. Due to the restriction of eight cardinal directions and problems with only $45^{\circ}$ and $90^{\circ}$ angles, there are 16 different problems. For each of the 16 problems it is possible to construct multiple different configurations and corresponding solutions, which all satisfy the premises. That is, the problems are under-determined. Using the example trial above, there are four possible different mental models, which are illustrated in Fig. 2.

\footnotetext{
${ }^{1}$ The language of the experiment was German. Tasks are translated into English here.
} 
Depending on the order of the named entities, there are multiple possibilities to present each problem. The example above could also be given as a) " $\mathrm{X}$ is southwest of Z" and "Y is east of X" or as $b$ ) " $Z$ is northeast of $X$ " and "Y is east of X", for example. As Bertel et al. (2010) found no indication that the order of the entities impacts the type of mental model that is constructed, the tasks of this study are only presented in one order, namely in the way described in b) above.

We employed a head-mounted SensoMotoric Instruments (SMI) iView X HED eye tracking system to record the subject's eye movements.

\subsection{Procedure}

The participating subjects sat on a chair at a table facing a blank white wall at a distance of approximately $1 \mathrm{~m}$. Subjects had to place their hands on their legs under a table holding a computer mouse in the one hand and a small ball in the other one. This prevented the subjects from using their fingers to solve the tasks. The eye tracker was fixed on the subject's head and calibrated. Subjects were told that the eye tracker's camera measures their pupil dilation in order to relate it to the difficulty of the tasks, i.e., they were not aware that their eye movements were recorded. All instructions of the experiment were projected on the white wall.

To make subjects familiar with the cardinal directions, the experiment started with a learning phase. Each task of the learning phase comprised an acoustically presented statement and an answer screen with a question. Each statement was triggered by the subject clicking her mouse and contained the direction relation between two entities; e.g., " $\mathrm{K}$ is northwest of U". After 4 seconds the answer screen appeared, which depicted the reference entity "U" surrounded by the numbers 1 to 8 in a counterclockwise circular order together with the question "As seen from U, where is K?". The eight numbers represented the eight cardinal directions $(1=$ north, 2 = northwest, $3=$ west, $\ldots 8=$ northeast). The participants answered by naming the number, which corresponded to the position of " $K$ " and the experimenter typed the number into the computer. Depending on whether the answer was correct, "correct" or "wrong" together with the correct direction were projected. The learning phase ended as soon as each of the eight cardinal directions had been recognized correctly twice in a row.

The experiment consisted of 32 problem trials. These consisted of two instances for each of the 16 possible problems. The instances differed in the letters used. In addition, 4 pre trials were presented in the beginning in order to familiarize the subjects with the tasks. To prevent memory effects due to the identical order of the letters within in the problem trials, 12 filler trials with a different order were mixed in randomly. Thus, participants had to work on 48 trials in total, whereas only the 32 actual problem trials were used in the analysis. The trials were presented in a randomized order. The subjects used the mouse to trigger the acoustic presentation of the first premise of each trial. As soon as the subjects understood the statement, they clicked again for the presentation of the second premise. Similarly, they triggered the acoustic presentation of the question after having processed the second premise. When the subject felt she found the answer, she clicked the mouse again for the projected answer screen to appear. The answer screen and the answer procedure were the same as in the training phase, except that the question was not projected on the screen. To continue to the next trial, the subject had to click again. Subjects were allowed to take breaks between the trials. 
The subject's eye movements were recorded during all tasks. When participants closed their eyes, the experimenter advised the subject to open her eyes again. The calibration, the learning phase and the test phase together typically lasted between 45 and 60 minutes.

\subsection{Analysis of the Eye Tracking Data}

In this section we will describe how the eye tracking data was analyzed to identify whether eye movements occurred along the given spatial relations between the entities for each trial. The same method of analysis was employed in both experiments. The raw eye tracking data collected by the iView X software was first converted using the IDF Event Detector to generate the list of fixations made by the subject. For this we had to set two parameters, maximum dispersion and maximum duration, which together define when a fixation is identified. Taking into account the distance between the subject and the white wall, we set these at 40 pts and $180 \mathrm{~ms}$ given a resolution of $752 \times 480$ of the tracked visual field. From the sequence and coordinates of the subject's fixations, the saccades were calculated automatically, as between two fixations at different locations, there must have been a saccade in order for the gaze to get from one point to the other.

Using the sequence of saccades, defined by the starting and ending coordinates, we classified each saccade into one of eight classes, which correspond to the eight cardinal directions, which were used in the trials: we uniformly mapped all possible angles of a saccade, which is basically a vector in a Cartesian plane and thus has an angle, to the cardinal directions. Each direction corresponds to a range of angles on a degree circle with each direction taking up $\left(360^{\circ} / 8\right)=45^{\circ}$. For example "north" corresponds to all angles in the range of $0^{\circ} \pm\left(45^{\circ} / 2\right)=0^{\circ} \pm 22,5^{\circ}=\left[337.5^{\circ} ; 22.5^{\circ}\right]$. It is to note that the eye movements classified in this way are relative eye movements, i.e., the absolute coordinates do not matter. This makes sense considering the fact that the subject moves her head during the trials and that also arbitrary eye movements occur in between. Given this classification of each saccade to one cardinal direction, we were able to investigate the relation between the given direction and observed eye movements during a trial.

We have so far only looked at the eye movements that occurred after the first premise of each problem trial was read. In particular, we analyzed only the eye movements that occurred in the second half of the time span between the first mention of the direction of the first premise and the time at which the subject clicks to indicate she has understood the premise and is ready for the second premise. We have discarded the first half of this time span, as we assume the subject needs to first process the presented information before she starts the construction of either a mental model or mental image.

As we expected eye movements to occur not only in the direction read, but also in the opposite one, we checked the set of saccades for both of these directions. Assuming a mental image of for example A being north of B, we should not only expect internal attention shifts from A to B but also from B to A during inspection as well as construction of the image. Thus, we always compare the absolute number of made saccades to the combined absolute number of saccades made along the given direction in the first premise and its opposite direction in the analysis of both experiments. 


\subsection{Results of Experiment 1}

\section{Accuracy}

Despite the reported high difficulty of the task, the overall error rate was relatively low with an average error of $12.4 \%$. The error rate ranged from $0 \%$ to $28.1 \%$. Answers were counted as errors if they did not match any of the possible directions given the premises. Only the correct answers are relevant for the investigation of preferences, thus every incorrect answer was excluded from the analysis.

\section{Eye movements}

The proportion of eye movements along the given direction of the first premise and its opposite direction ranged from $14.29 \%$ to $34.88 \%$ with an average of $25.38 \%$ over all subjects. Assuming the directions given in the tasks do not have any influence on the eye movements, one would expect the eye movements being equally distributed over the eight cardinal directions, that is a proportion of $12.5 \%$ for each direction or $25 \%$ for the combination of a direction and its opposite direction. Given our average result of $25.38 \%$, there are not substantially more eye movements according to the named directions and its opposites. Binomial tests showed that only 1 subject out of 25 (see Fig. 4) made significantly ${ }^{2}$ more eye movements along the directions of the first premise. None of the other subjects moved their eyes significantly more often along the given directions. This is what one would expect, if - as hypothesized - eye movements do not reflect the reasoning process.

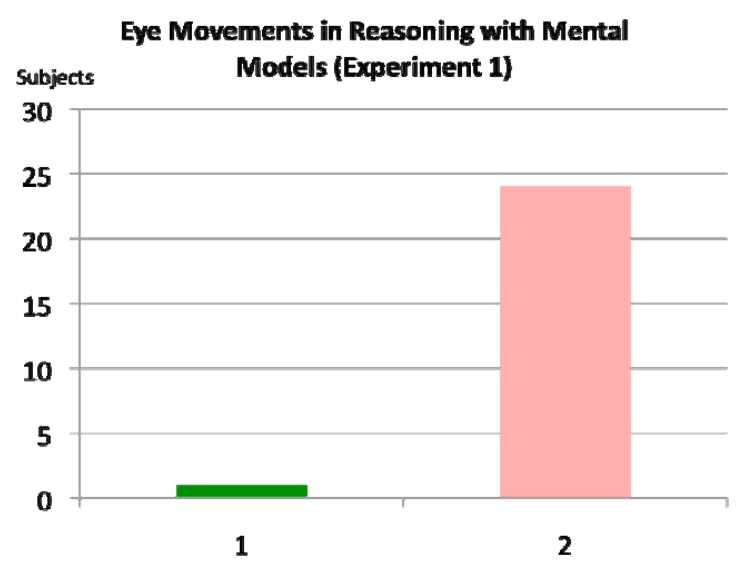

Fig. 4. The number of subjects that made significantly more eye movements along the directions given in the first premises and their opposites (1) and those that did not (2) during experiment 1

\section{Preferred mental models}

Every answer was classified as either being consistent or not consistent with the hypothesized preferred mental models (see section 1.2). For each subject the percentages

\footnotetext{
${ }^{2}$ If not stated otherwise, the level of significance is $\mathrm{p}=0.05$.
} 
of how often the subject constructed the hypothesized preferred or other mental models were computed. These percentages served as the basis for subsequent analyses. Since percentages are often not distributed normally, non-parametric test were employed whenever this was necessary and possible.

If there were no preferences, the probability of a subject choosing any possible correct answer would be uniform. For each $45^{\circ}$ problem, the hypothesized preferred solutions should then be constructed on $25 \%$ of all trials, as there are four correct answers. For the $90^{\circ}$ problems, there are three different correct answers, including the hypothesized preferred one. That is, each solution should be chosen on $33 \%$ of the trials, given there are no preferences.

Regarding the $90^{\circ}$ problems, the hypothesized preferred models were chosen on $88 \%$ of all trials which indicates a strong preference for these models (see Fig. 5). Indeed, the preference is much stronger than one would expect by chance $\left(t_{(24)}=17,551\right.$; $p<0.001)$. We can conclude, that the results confirm the hypothesis regarding preferred mental models for the $90^{\circ}$ problems.
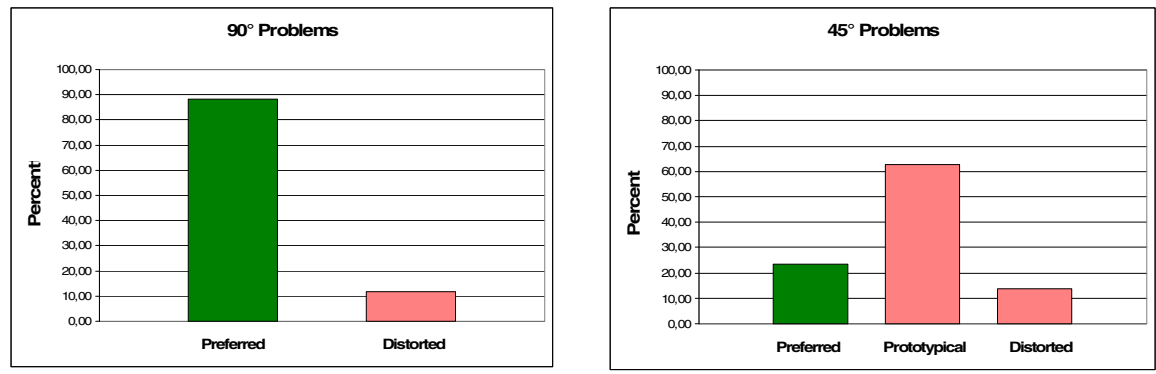

Fig. 5. Average percentages of trials on which subjects constructed the hypothesized preferred mental models compared to those that constructed other correct models for the $90^{\circ}$ and the $45^{\circ}$ problems of experiment 1

Regarding $45^{\circ}$ problems, the preferred, prototypical, and distorted models were chosen on $23 \%, 63 \%$, and $14 \%$ of the trials, respectively. Statistical analyses revealed that subjects exhibited no preference for the hypothesized preferred models $\left(t_{(24)}=-\right.$ $1.021 ; p>0.3)$, a significant preference for prototypical models $\left(t_{(24)}=5.352 ; p<\right.$ $0.001)$, and a significant dispreference for distorted models $\left(t_{(24)}=-9.995 ; p<0.001\right)$. The strong preference for prototypical models is further corroborated by pairwise comparisons using the Wilcoxon test: Prototypical models are chosen more frequently than both preferred $(z=-2.145 ; p<0.05)$ and distorted $(z=-3.704 ; p<0.001)$ models while frequencies for preferred and distorted models do not differ significantly from each other $(z=-0.392 ; p>0.65)$. Concluding, the results do not confirm the hypothesis regarding preferred mental models for the $45^{\circ}$ problems. Thus, results of Bertel et al. (2010) regarding the $45^{\circ}$ problems could not be replicated. This may be due to the different experimental settings. Subjects in Bertel et al. (2010) were presented the premises and questions on a monitor whereas in our experiments they were facing a blank white wall and had to keep their hands below the table while the premises were presented acoustically. 


\section{Experiment 2 - Reasoning with Mental Images}

In this experiment we induced the use of mental imagery for reasoning about cardinal directions. We assume that by using stimuli, that are easier to visualize, subjects will employ visual representations to solve the tasks, i.e., visual mental images. We hypothesize, that during a reasoning process based on mental images, significantly more eye movements will occur along the cardinal directions given in the tasks than along the other directions. Besides the minor modification of the instructions, this experiment is identical to the first one and reused the reasoning problems as well as the hypotheses regarding preferred mental models of experiment 1.

\subsection{Participants and Materials}

23 undergraduate students of the University of Bremen, 12 male and 11 female, participated in the study for monetary compensation. The stimulus material used in this experiment is the same as in experiment 1 and is described in section 2.1.

\subsection{Procedure}

The procedure of this experiment resembles that of experiment 1 (see section 2.2). The only difference is a slight change in the instructions given to the participants. In contrast to experiment 1 , the subjects were told that the entities in the experimental tasks are meant to be cities on a map. Furthermore, they were told that each city is marked on the map as a little red square with the according letter written next to it labeling the city. Subjects were instructed to imagine the given constellation of the three cities on the imaginary map to solve the reasoning problem. These instructions were used for the learning phase as well as for the test phase. By describing the stimuli in a more visual way than in the first experiment, we wanted to induce the use of mental imagery. The acoustic presentation of the stimuli during the trials remained unchanged.

\subsection{Results of Experiment 2}

Accuracy

In this experiment, the error rate ranged from $0 \%$ to $28.1 \%$ with an average of $9.5 \%$.

\section{Eye movements}

We proceeded as in the previous experiment, described in section 2.3 and 2.4. We related the proportion of eye movements that occurred according to the cardinal directions given in the premises and their corresponding opposite directions, which was $30.46 \%$ (with a range from $16.46 \%$ to $50.40 \%$ ) over all subjects, to the level of chance, namely $25 \%$. Binomial tests showed that 10 participants made significantly more eye movements along the expected directions than along the other directions, whereas 13 subjects did not (see Fig. 6). This number of 10 participants is significantly more than expected, given the $\mathrm{p}=0.05$ probability of error when testing the individual subject. These results confirm our hypothesis about the correlation between eye movements and the spatial relations in reasoning with visual mental images. 


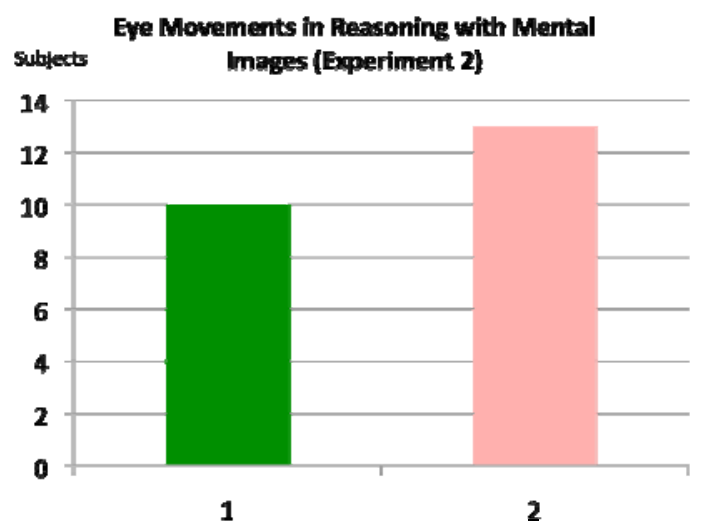

Fig. 6. Comparison of subjects that made significantly more eye movements along the directions in the premises (1) and those that did not (2) during experiment 2

\section{Preferred mental models}

On average, 93\% hypothesized preferred mental models were constructed for $90^{\circ}$ problems (see Fig. 7). As in experiment 1, the preference is much stronger than one would expect if participants had no preferences $\left(t_{(22)}=29,846 ; p<0.001\right)$.

Regarding the $45^{\circ}$ problems, the preferred, prototypical, and distorted models were chosen on $48 \%, 46 \%$, and $6 \%$ of the trials, respectively. Statistical analyses revealed that all these percentages differed significantly from percentages expected if people had no preferences $\left(t_{(22)}=2.683 ; p<0.05 ; t_{(22)}=2.512 ; p<0.05 ; t_{(22)}=-25.36 ; p<\right.$ 0.001 for preferred, prototypical, and distorted models, respectively). Thus, participants exhibited marked preferences for both preferred and prototypical models. Pairwise comparisons using the Wilcoxon test indicate that there is no difference in preference for preferred and prototypical models $(z=-0.305 ; p>0.75)$, while both of these are chosen significantly more frequently than distorted models $(z=-3.569 ; p<$ 0.001 and $z=-3.262 ; p<0.001$, respectively). Consequently, the results of Bertel
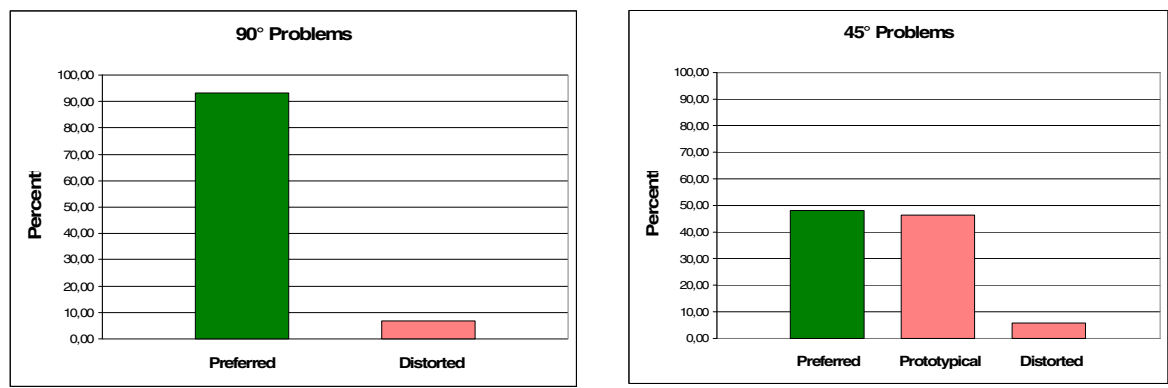

Fig. 7. Average percentages of trials on which subjects constructed the hypothesized preferred mental models compared to those that constructed other correct models for the $90^{\circ}$ and the $45^{\circ}$ problems of experiment 2 
et al. (2010) were largely replicated, showing a reliable preference for the hypothesized preferred mental models.

\section{Comparing Eye Movements and Preferred Mental Models in Experiment 1 and 2}

One main goal of this investigation was to explore the occurrence of eye movements in reasoning with cardinal directions based on mental models and mental images. We hypothesized that eye movements according to the spatial relations of the tasks will occur when employing visual mental images (as in experiment 2) and that this will not be the case when employing spatial mental models (as in experiment 1). Results show that in the mental image condition $30.56 \%$ of the eye movements occurred according to the directions and respective opposite directions of the tasks (see section 3.3). This is well above the level of change of $25 \%$. In contrast, the proportion of eye movements in line with the directions of the premise when using spatial mental models was considerably lower with only $25.38 \%$, which equals the level of chance (see section 2.4). This comparison already indicates an influence of the representation format on eye movements during the tasks.

In addition, as described in section 3.3 , there are significantly more subjects $(10$ out of 23) in the mental imagery experiment that showed significantly more eye movements along the directions in the task. In contrast only 1 subject out of 25 (see section 2.4) showed significantly more eye movements along the given directions during the mental model experiment. A $\chi^{2}$ test showed that this difference between the two experiments is significant $(\mathrm{p}<0.01)$. This means that there is a clear influence of the used mental representation on eye movements during spatial reasoning tasks.

One could think of an alternative explanation for the different eye movement patterns in the two experiments. The change in instruction could have led the subjects to use a different perspective for the two conditions. Specifically, subjects could have used an egocentric perspective for solving the tasks of the first experiment and a survey perspective for the second one, for which the image of a map is suggested in the instructions. ${ }^{3}$ We do, however, assume no change in perspective between the two conditions, as subjects are trained to apply a survey perspective in both cases as described in section 2.2.

Regarding the preferred mental models, both experimental conditions yield similar results for the $90^{\circ}$ problems, but there is a notable difference for the $45^{\circ}$ problems. Our hypothesized preference for those problems seems to only apply when mental imagery is used to solve the tasks. In the mental model condition the prototypical model was actually constructed significantly more often than the other models. This indicates that the preferred mental model may depend on the underlying mental representation that is used.

\section{Conclusions}

This contribution reports two experiments that investigated human spatial reasoning about cardinal directions. Of particular interest was the influence the representation

\footnotetext{
${ }^{3}$ We thank one of the reviewers for pointing this out.
} 
format employed for reasoning has on the eye movements during reasoning. The experimental results have a number of important implications. First, the existence of preferences in both experiments (a) further corroborates the idea that preferences are robust and pervasive phenomena in human spatial reasoning and (b) suggests that preferences also occur when the employed representations are more image-like (i.e., preferred mental images). Second, the fact that the type of preferences observed in the present studies partly differ from previously observed preferences in reasoning about cardinal directions suggests an unanticipated susceptibility of the type of preferences to procedural details. Third, when employing an abstract representation format such as spatial mental models, eye movements do not reflect the spatial layout of the represented situation. This is in contrast to eye movements observed in the scope of employing more visual representations: when more visual representations such as mental images are used, eye movements often reflect the spatial relations that are represented. Fourth, the differing impact that visual and abstract representations have on eye movements opens up the possibility to utilize observed eye movements as evidence for the type of representation currently employed by a person. This could be of advantage in building or improving computer-based assistance systems that support a human reasoner in solving spatial problems such as, for instance in spatial planning or in architectural design. If the assistance system receives evidence about the format in which the reasoner currently represents the task-relevant knowledge, the system will be able to better predict the cognitive state of the reasoner (e.g., based on an available cognitive model) and, thus, will be able to provide better assistance.

\section{Acknowledgements}

This paper presents work done in the project R1-[ImageSpace] of the Transregional Collaborative Research Center SFB/TR 8 Spatial Cognition. Funding by the German Research Foundation (DFG) is gratefully acknowledged. We also thank Sven Bertel for his suggestions regarding experimental design.

\section{References}

Bertel, S., Schultheis, H., Barkowsky, T.: Modeling mental spatial reasoning about cardinal directions. Manuscript submitted for publication (2010)

Brandt, S.A., Stark, L.W.: Spontaneous eye movements during visual imagery reflect the content of the visual scene. Journal of Cognitive Neuroscience 9(1), 27-38 (1997)

Brockmole, J.R., Wang, R.F.: Switching between environmental representations in memory. Cognition 83, 295-316 (2002)

Demarais, A.M., Cohen, B.H.: Evidence for image-scannig eye movements during transitive inference. Biological Psychology 49, 229-247 (1998)

Finke, R.: Principles of mental imagery. MIT-Press, Cambridge (1989)

Frank, A.U.: Qualitative spatial reasoning: Cardinal directions as an example. International Journal of Geographical Information Science 10(3), 269-290 (1996)

Jahn, G., Johnson-Laird, P.N., Knauff, M.: Reasoning about consistency with spatial mental models: Hidden and obvious indeterminacy in spatial descriptions. In: Freksa, C., Knauff, M., Krieg-Brückner, B., Nebel, B., Barkowsky, T. (eds.) Spatial Cognition IV. LNCS (LNAI), vol. 3343, pp. 165-180. Springer, Heidelberg (2005) 
Johansson, R., Holsanova, J., Holmqvist, K.: Pictures and spoken descriptions elicit similar eye movements during mental imagery, both in light and in complete darkness. Cognitive Science 30, 1053-1079 (2006)

Johnson-Laird, P.N.: The three-term series problem. Cognition 1, 57-82 (1972)

Johnson-Laird, P.N.: Mental models. Harvard University Press, Cambridge (1983)

Johnson-Laird, P.N.: Mental models. In: Posner, M.I. (ed.) Foundations of cognitive science, pp. 469-499. MIT-Press, Cambridge (1989)

Knauff, M.: A neuro-cognitive theory of deductive relational reasoning with mental models and visual images. Spatial Cognition \& Computation 9, 109-137 (2009)

Knauff, M., Rauh, R., Schlieder, C.: Preferred mental models in qualitative spatial reasoning: A cognitive assessment of Allen's calculus. In: Proceedings of the Seventeenth Annual Conference of the Cognitive Science Society, pp. 200-205. Erlbaum, Mahwah (1995)

Kosslyn, S.M.: Image and Brain: The Resolution of the Imagery Debate. Harvard University Press, Cambridge (1994)

Kosslyn, M.S., Alpert, M.N., Thompson, L.W., Maljkovic, V., Weise, B.S., Chabris, F., et al.: Visual mental imagery activates topographically organized visual cortex: PET investigations. Journal of Cognitive Neuroscience 5, 263-287 (1993)

Kosslyn, M.S., Thompson, L.W.: When is early visual cortex activated during visual mental imagery? Psychological Bulletin 129(5), 723-746 (2003)

Laeng, B., Teodorescu, D.-S.: Eye scanpaths during visual imagery reenact those of perception of the same visual scene. Cognitive Science 26, 207-231 (2001)

Ragni, M., Fangmeier, T., Bittner, A., Konieczny, L.: Incremental model construction: Eyemovements reflect mental representations and operations - even if there is nothing to look at. In: Proceedings of the 31st Annual Conference of the Cognitive Science Society, pp. 3046-3051. Cognitive Science Society, Austin (2009)

Schultheis, H., Bertel, S., Barkowsky, T., Seifert, I.: The spatial and the visual in mental spatial reasoning: An ill-posed distinction. In: Barkowsky, T., Knauff, M., Ligozat, G., Montello, R.D. (eds.) Spatial Cognition 2007. LNCS (LNAI), vol. 4387, pp. 191-209. Springer, Heidelberg (2007)

Shepherd, M., Findlay, J.M., Hockey, R.J.: The relationship between eye movements and spatial attention. Quarterly Journal of Experimental Psychology 38A, 475-491 (1986)

Sloman, A.: Afterthoughts on analogical representation. In: Proc. Theoretical Issues in Natural Language Processing (TINLAP-1), Cambridge, MA, pp. 164-168 (1975)

Spivey, M.J., Geng, J.J.: Oculomotor mechanisms activated by imagery and memory: eye movements to absent objects. Psychological Research 65, 235-241 (2001)

Tversky, B.: Cognitive maps, cognitive collages, and spatial mental models. In: Frank, A.U., Campari, I. (eds.) COSIT 1993. LNCS, vol. 716, pp. 14-24. Springer, Heidelberg (1993) 